\title{
Generalized Variance-Ratio Tests in the Presence of Statistical Dependence
}

ARTICLE in JOURNAL OF TIME SERIES ANALYSIS · JANUARY 2006

Impact Factor: $0.81 \cdot$ DOI: $10.1111 /$ jtsa.12124 - Source: RePEC

3 AUTHORS, INCLUDING:

Periklis Kougoulis

Abu Dhabi University

4 PUBLICATIONS 6 CITATIONS

SEE PROFILE
Jerry Coakley

University of Essex

91 PUBLICATIONS 1,161 CITATIONS

SEE PROFILE 


\title{
Generalized variance ratio tests in the presence of statistical dependence
}

\author{
Jerry Coakley, Periklis Kougoulis and John C. Nankervis* \\ Department of Accounting, Finance and Management and Essex Finance Centre \\ University of Essex
}

February 15, 2006

\begin{abstract}
We develop extensions of the variance-ratio statistic for testing the hypothesis a time series is uncorrelated and investigate their finite-sample performance. The tests employ an estimator of the asymptotic covariance matrix of the sample autocorrelations that is consistent under the null for general classes of innovations including EGARCH and non-MDS processes. Monte Carlo experiments show that our tests have better finitesample size and power properties than the standard variance-ratio tests in experiments using time series generated by EGARCH and non-MDS processes.
\end{abstract}

Keywords: Variance-Ratio statistic; Non-MDS process; Monte Carlo JEL classification: C22, C14, C15, G10

\footnotetext{
${ }^{*}$ We would like to thank Stylianos Bounanos and Adrian Clark for providing access to the VASE Laboratory's cluster machines at the Electronic Systems Engineering Department of the University of Essex. Kougoulis is grateful for financial support provided by the Greek State Scholarship Foundation (IKY). Corresponding author: John C. Nankervis, Department of Accounting, Finance and Management, University of Essex, Wivenhoe Park, Cochester C04 3SQ. Tel +44 1206 873973. Fax +44 1206 873429. E-mail: jcnank@essex.ac.uk.
} 


\section{Introduction}

The variance-ratio (VR) statistic has been widely used for testing the hypothesis that financial or economic time series follow a random walk. It has been popularized by the work of Lo and MacKinlay (1988). If a series $\left\{x_{t}\right\}_{-\infty}^{\infty}$ follows a random walk, the first differences $y_{t}=x_{t}-x_{t-1}$ are independent (and hence also uncorrelated) for all lags and the series $x_{t}$ is said to belong to the class of martingale difference sequences (MDS). The VR statistic exploits the fact that the increments of a random walk - here $y_{t}$ - are by definition stationary and uncorrelated at all lags. The variance-ratio statistic for aggregation value $q[\operatorname{VR}(q)]$ is defined as the variance of the $q$-th period difference $x_{t}-x_{t-q}$ over $q$ times the variance of the one-period or first difference $y_{t}=x_{t}-x_{t-1}$. It converges to 1 under the null that $x_{t}$ is a random walk.

The essence of whether a time series is a random walk is related to its predictability. Strictly speaking, when a series follows a random walk model, the increments are independently distributed and thus are unpredictable. The VR statistic can be thought of as a test of the unforecastability of the first difference of the time series under study. ${ }^{1}$ The importance of the unforecastability of finacial time series has lead to the development of VR tests under weaker conditions of statistical dependence. In particular, the first differences of the series can be uncorrelated rather than independent and do not have to be Gaussian. For example, the increments can be uncorrelated but statistically dependent and conditionally heteroskedastic.

Lo and MacKinlay (1988) derive the asymptotic distribution of the VR statistic for the following two cases: (i) the increments are independent and identically Gaussian distributed and (ii) the increments are uncorrelated but exhibit conditional heteroskedasticity of a particular form. ${ }^{2}$ The basis of their derivation stems from the observation that the $\operatorname{VR}(q)$ statistic can be expressed as a weighted sum of the sample autocorrelations. Asymptotically valid tests based on the VR statistic can be obtained from consistent estimates of the asymptotic covariance matrix of the sample autocorrelations. The asymptotic covariance matrix of the sample autocorrelations is an identity matrix when the increments are independent and identically distributed and under case (ii) above considered by Lo and MacKinlay the asymptotic variance of the sample autocorrelations takes the form of a diagonal matrix. Guo and Phillips (1998) have proposed a consistent estimator of the asymptotic covariance matrix of the sample autocorrelations for the case of general MDS processes. This asymptotic variance-covariance matrix is, in general, nondiagonal in the latter case.

The Guo and Phillips covariance matrix is more general in the sense that it

\footnotetext{
${ }^{1}$ The forecastability of a series (i.e. stock prices) is usually related to tests of the Efficient Market Hypotheses (EMH). Although predictability is a necessary condition for the rejection of the EMH, it is by no means sufficient.

${ }^{2}$ While Lo and MacKinlay consider the asymptotic distribution when the sample size $n \rightarrow$ $\infty$, Richardson and Watson (1989) derive the asymptotic distribution when $n \rightarrow \infty, q \rightarrow \infty$ and $q / n \rightarrow \delta>0$. More importantly, Chen and Deo (2004) derive the asymptotic distribution when $n \rightarrow \infty, q \rightarrow \infty$ and $q / n \rightarrow 0$. The latter case is to be preferred when considering tests over longer horizons or when $q$ is relatively large compared to the sample size $n$.
} 
allows for extensions to standard GARCH processes to account for asymmetry in the unconditional returns, known as leverage effects (Zakoian 1994, Nelson 1991, Ding, Granger and Engle 1993). Another approach in the attempt to fully capture the high kurtosis and the asymmetry of the unconditional returns is to model volatility clustering via GARCH models with skewed innovations. For example Hansen (1994) suggested a skewed Student distribution while Fernández and Steel (1998) suggested another skewed type distribution that was applied under the GARCH framework by Lambert and Laurent (2001).

In this paper generalized variance-ratio statistics are developed by adopting an approach similar to the extension of the Box-Pierce statistic by Lobato, Nankervis and Savin (2002). Like the Box-Pierce statistic, the VR statistic can be expressed as a functional of the sample autocorrelations. The Box-Pierce statistic is the sample size times the sum of the squares of the sample autocorrelations. The Box-Pierce statistic has been developed both for a general class of MDS processes as well as for non-MDS processes. Lobato et al. suggested a statistic which is in the spirit of a Lagrange multiplier because it is based on a consistent estimator of the asymptotic covariance matrix of the sample autocorrelation under the null. The extension of the Box-Pierce statistic is based on a consistent nonparametric estimator of the variance-covariance matrix of the sample autocorrelations and is therefore applicable to the construction of an extended variance-ratio statistic.

The finite sample performance of the VR statistic is assessed by Monte Carlo experiments. The standard versions of the VR statistics, as suggested by Lo and MacKinlay are compared relative to the alternative extensions. In order to evaluate the size of the different VR versions, we consider examples of uncorrelated series when the data generating process is either a martingale difference sequence (MDS) or a non-martingale difference sequence (non-MDS). In particular, we consider the data generating process to be either a $\operatorname{GARCH}(1,1)$ or an $\operatorname{EGARCH}(1,1)$ for the MDS case. Non-MDS examples include uncorrelated time series, such as the bilinear model of Granger and Andersen(1978) or the nonlinear moving average model of Tong (1990). The power of the test is examined by considering the DGP of the increments to be either an autoregressive process of first or sixth order, or alternatively a first or a second order moving average process, where the errors follow the processes used for the corresponding size evaluation.

The paper is organized as follows. Section 2 introduces the necessary notation and assumptions while Section 3 develops the extensions of the varianceratio statistic. The results of the Monte Carlo experiments regarding the size of the variance ratio are reported in Section 4 while the corresponding results for the power of the test are presented in Section 5. Section 6 concludes. 


\section{Preliminaries}

Suppose that the hypothesis to be tested is that the series $\left\{x_{t}\right\}$ follows a random walk with $\mathrm{drift}^{3}$. The essence of testing this hypothesis is the unforecastability of the $x_{t}$ series and therefore the restrictive assumption that the errors are i.i.d. can be relaxed. Define the real valued covariance stationary time series $y_{t}=\Delta x_{t}=$ $x_{t}-x_{t-1}$ with mean $\mu$ and square integrable spectrum. Let $\widehat{\mu}=(1 / T) \sum_{t=1}^{T} y_{t}$ denote the sample mean of the series $y_{t}$ for a sample of size $T$. The lag- $j$ autocorrelation of $y_{t}$ is defined as $\gamma(j)=E\left[\left(y_{t}-\mu\right)\left(y_{t-j}-\mu\right)\right]$, while the lag- $j$ autocovariance of $y_{t}$ is $\rho(j)=\gamma(j) / \gamma(0)$. The sample analogues of the lag- $j$ autocovariance and autocorrelation are denoted as $\widehat{\gamma}(j)=(1 / T) \sum\left(y_{t}-\widehat{\mu}\right)\left(y_{t-j}-\widehat{\mu}\right)$ and $r(j)=\widehat{\gamma}(j) / \widehat{\gamma}(0)$, respectively. The vector of the sample autocovariances is denoted by $\widehat{\gamma}=[\widehat{\gamma}(0), \widehat{\gamma}(1), \ldots, \widehat{\gamma}(K)]^{\prime}$ where $K$ is the highest lag considered. Similarly, the vector of sample autocovariances is $r=[r(1), \ldots r(K)]^{\prime}$. The vectors of population autocorrelations and population autocovariances are denoted as $\gamma=[\gamma(0), \gamma(1), \ldots, \gamma(K)]^{\prime}$ and $\rho=[\rho(1), \ldots \rho(K)]^{\prime}$ respectively.

The process $y_{t}$ is assumed to be a weakly dependent process for which the vector of sample autocovariances satisfies

$$
\sqrt{T}(\widehat{\gamma}-\gamma) \Rightarrow N(0, C)
$$

The matrix $\mathrm{C}$ is assumed to be finite and positive definite. $\mathrm{C}$ is $2 \pi$ times the spectral density matrix at zero frequency of the vector $\omega_{t}$. The later is defined as $\omega_{t}=\left[\omega_{1, t}, \omega_{2, t}, \ldots \omega_{K, t}\right]^{\prime}$ where $\omega_{k, t}=\left(y_{t}-\mu\right)\left(y_{t-k}-\mu\right)$ for all $k=1,2, \ldots K$.

The $i j$ th component of the matrix $\mathrm{C}$ is given by:

$$
\begin{aligned}
c_{i j}= & \sum_{d=-\infty}^{d=+\infty}\left\{E ( y _ { t } - \mu ) \left(\left(y_{t-i}-\mu\right)\left(y_{t+d}-\mu\right)\left(y_{t+d-j}-\mu\right)\right.\right. \\
\text { for } i, j= & \left.-E\left(y_{t}-\mu\right)\left(y_{t-i}-\mu\right) E\left(y_{t+d}-\mu\right)\left(y_{t+d-j}-\mu\right)\right\} \\
& 0,1, \ldots K
\end{aligned}
$$

Applying the delta method to equation (1) it can be shown that the sample autocovariances converge asymptotically to $\mathrm{N}(0, \mathrm{~V})$.

$$
\sqrt{T}(r-\rho) \Rightarrow N(0, V)
$$

where the $i j$ th component of $\mathrm{V}$ is given by

$$
v_{i, j}=\gamma(0)^{-2}\left[c_{i, j}-\rho(i) c_{0 j}-\rho(j) c_{0 i}+\rho(i) \rho(j) c_{00}\right.
$$

\footnotetext{
${ }^{3}$ Under the null that $x_{t}$ follows a random walk with drift $\mu$ we have,

$$
x_{t}=\mu+x_{t-1}+u_{t}
$$

where the $u_{t} \sim I N(0,1)$.
} 


\section{$3 \quad$ Existing and new $\operatorname{VR}(q)$ tests}

In the first part of this section the main fomulae regarding the variance-ratio test are briefly presented. A detailed exposition of the variance-ratio statistic can be found in Lo\&MacKinlay $(1988,1989)$. Our extensions of the $\operatorname{VR}(q)$ test are developed in the second part.

\subsection{Existing $\operatorname{VR}(q)$ test}

The maintained (null) hypothesis can be described by the following relationship:

$$
x_{t}=\mu+x_{t-1}+u_{t}
$$

where $\mu$ is an arbitrary drift parameter and $u_{t}$ is a zero mean random disturbance term with variance $E\left(u_{t}^{2}\right)=\sigma_{u}^{2}$ and $E\left(u_{t} u_{t-j}\right)=0$ for all $t, j$. Equation (3) reduces to the traditional random walk model by imposing the additional restriction that the $u_{t}$ are i.i.d.. However, this stronger assumption can be relaxed and the VR test can be studied under more general conditions of statistical dependence.

The VR statistic is based on the fact that the variance of the increments $u_{t}$ is linear in the observation interval. For example, under the null, the variance of the $q$ th differences of $x_{t}-\left(x_{t}-x_{t-q}\right)$ - is $q$ times the variance of the first differences $\left(x_{t}-x_{t-1}\right)$. The scalar $q$ is known as the aggregation value.

The overlapping adjusted variance-ratio statistic for aggregation value $q$ and for sample size of $T+1=n q+1$ observations of $x_{t}$ is defined as:

$$
V R(q)=\frac{\sum_{t=q}^{T}\left[x_{t}-x_{t-q}-q \widehat{\mu}\right]^{2}}{\sum_{t=1}^{T}\left[x_{t}-x_{t-1}-\widehat{\mu}\right]^{2}} * \frac{(T-1)}{q(T-q+1)\left(1-\frac{q}{T}\right)}
$$

By noting that the $q$ th difference of $x_{t}$ is

$$
x_{t}-x_{t-q}=\sum_{s=0}^{q-1} y_{t-s}
$$

equation(4) can be rewritten as

$$
V R(q)=\frac{\sum_{t=q}^{T}\left[\sum_{s=0}^{q-1}\left(y_{t-s}\right)-q \widehat{\mu}\right]^{2}}{\sum_{t=1}^{T}\left[y_{t}-\widehat{\mu}\right]^{2}} * \frac{(T-1)}{q(T-q+1)\left(1-\frac{q}{T}\right)}
$$

\subsection{Extending the VR test}

The $\operatorname{VR}(q)$ statistic for aggregation value $q$, can be shown to be approximately a linear combination of the first $q-1$ autocorrelation coefficient estimators of the first differences of $x_{t}$, the $y_{t}$, with arithmetically declining weights (Lo and MacKinlay 1988, 1989).

In particular we have 


$$
V R(q) \simeq 1+\frac{2(q-1)}{q} r(1)+\frac{2(q-2)}{q} r(2)+\ldots+\frac{2}{q} r(q-1)
$$

Denoting the $(q-1) \times 1$ vector of the weights as $w=\left[\frac{2(q-1)}{q}, \frac{2(q-2)}{q}, \ldots, \frac{2}{q}\right]^{\prime}$ and the centered variance ratio as $V R_{c}(q)=V R(q)-1$, equation (5) can be expressed as $^{4}$ :

$$
V R_{c}(q) \simeq w^{\prime} r
$$

where $r=[r(1), \ldots r(q-1)]^{\prime}$ is the vector of sample autocorrelations of the series $y_{t}$ up to order $q-1$.

Recalling that $\sqrt{T}(r-\rho) \Rightarrow N(0, V)$ by equation (2) and with respect to $V R_{c}(q) \simeq w^{\prime} r$ the asymptotic distribution of the VR statistic can be obtained. In particular

$$
\sqrt{T} w^{\prime}(r-\rho) \Rightarrow N\left(0, w^{\prime} V w\right)
$$

which after normalization reduces to

$$
\sqrt{T}\left[w^{\prime}(r-\rho)\right]\left(w^{\prime} V w\right)^{-\frac{1}{2}} \Rightarrow N(0,1)
$$

where $V$ is the $(q-1) \times(q-1)$ asymptotic variance-covariance matrix of the autocovariances. It is then obvious that the centered variance-ratio statistic follows the standard normal distribution. In particular by equation (5.1) and (6.1) we have:

$$
\sqrt{T *} V R_{c}(q) *\left(w^{\prime} V w\right)^{-\frac{1}{2}} \Rightarrow N(0,1)
$$

A feasible test for the VR statistic can be obtained either by replacing $V$ by a known matrix or by estimating $V$. More specifically, we propose a test based on the form $\sqrt{T} V R_{c}(q)\left(w^{\prime} \widehat{\widetilde{V}} w\right)^{-\frac{1}{2}}$ where $\widehat{\widetilde{V}}$ is a consistent estimator of $\widetilde{V}$ under the null. Lo and MacKinlay replace the $V$ matrix with the identity matrix when the first difference of the $x_{t}$ series $-\left\{y_{t}\right\}$ - is independent normally distributed. ${ }^{5}$

The extension of the $\operatorname{VR}(\mathrm{q})$ we propose is based on a consistent estimator of $V$ under the null $\left(H_{0}: \rho=\mathbf{0}\right)$. The estimation of $V$ by exploiting the restrictions imposed by the null is in the spirit of the Lagrange multiplier (LM) test. Under the null, the first differences of $x_{t}$ are uncorrelated so that the matrix $V$ simplifies to $\widetilde{V}=\left\{\gamma(0)^{-2} \widetilde{C}\right\}$ where $\widetilde{C}$ has its $i j$ th element

$$
\widetilde{c}_{i j}=\sum_{d=-\infty}^{d=\infty} E\left(y_{t}-\mu\right)\left(y_{t-i}-\mu\right)\left(y_{t+d}-\mu\right)\left(y_{t+d-j}-\mu\right) ; \quad i . j=1, \ldots q-1
$$

\footnotetext{
${ }^{4}$ We denote the centered overlapping adjusted variance ratio as $V R_{c}(q)$. Note, however that Lo and MacKinlay denote the same statistic as $\overline{M r}(q)$.

${ }^{5}$ In particular when $y_{t}=x_{t}-x_{t-1}$ is i.i.d. normal, $\rho=0$ and the null that the $x_{t}$ series is a random walk can be tested by $\sqrt{T} V R_{c}(q)\left(\frac{2(2 q-1)(q-1)}{3 q}\right)^{-1 / 2 \stackrel{a}{\sim}} N(0,1)$
} 
For MDS processes the only possible nonzero elements of $\widetilde{C}$ occur at $d=0$ and have the form $E\left(y_{t}-\mu\right)^{2}\left(y_{t-i}-\mu\right)\left(y_{t-j}-\mu\right)$. The $i j$ th element of $\widetilde{C}$ for MDS processes reduces to

$$
\widetilde{c}_{i j}=E\left(y_{t}-\mu\right)^{2}\left(y_{t-i}-\mu\right)\left(y_{t-j}-\mu\right) ; \quad i, j=1, . ., q-1
$$

Guo and Phillips (1998, Theorem 5) have developed a test, the $\mathrm{GP}_{k}$-test for uncorrelatedness up to order $K$ for the MDS case. Assuming that we have a MDS process, then we have a special case of the $\widetilde{V R}(q)$ test where $\widetilde{c}_{i j}$ is replaced by the sample analogue of $E\left(y_{t}-\mu\right)^{2}\left(y_{t-i}-\mu\right)\left(y_{t-j}-\mu\right)$. For some particular MDS processes, the test can be specialized further by assuming that $\widetilde{c}_{i j} \neq 0$ only when $d=0$ and $i=j$. In this case $\widetilde{V}$ is a diagonal matrix and is denoted by $V^{*}$ with

$$
c_{j j}^{*}=E\left(y_{t}-\mu\right)^{2}\left(y_{t-j}-\mu\right)^{2}, \quad j=1, . ., q-1
$$

and the diagonal elements of $V^{*}$ are $v_{j j}^{*}=\frac{c_{j j}^{*}}{\gamma(0)^{2}}$. The version of the VR test when the asymptotic covariance matrix is diagonal was introduced by Lo and MacKinlay (1988) and has been widely used in the literature when the $y_{t}$ series is assumed to exhibit conditional heteroskedasticity.

For the general case - when $y_{t}$ is either an MDS or a non-MDS process - an estimator of $\widetilde{V}$ is required. A consistent estimator can be obtained by estimating $\gamma(0)$ with $\widehat{\gamma}(0)$ and a nonparametric (or semi-parametric) estimate of the matrix $\widetilde{C}$. Under the null, the matrix $\widetilde{C}$ is the spectral density at zero frequency of the $(q-1)$ - dimensional vector process $\omega_{t}$. Therefore a consistent time-domain estimator of $\widetilde{C}$ is given by

$$
\widehat{\widetilde{C}}=\sum_{j} k\left(\frac{j}{\ell}\right) g(j)=\frac{1}{T} \sum_{j} \sum_{t} k\left(\frac{j}{\ell}\right) \widehat{\omega}_{t} \widehat{\omega}_{t-j}^{\prime}
$$

where $g(j)=\frac{1}{T} \sum_{t} k\left(\frac{j}{\ell}\right) \widehat{\omega}_{t} \widehat{\omega}_{t-j}^{\prime}$ with $\widehat{\omega}_{t}=\left[\widehat{\omega}_{1, t}, \widehat{\omega}_{2, t}, \ldots \widehat{\omega}_{q-1, t}\right]^{\prime}$ where $\widehat{\omega}_{k, t}=$ $\left(y_{t}-\widehat{\mu}\right)\left(y_{t-k}-\widehat{\mu}\right)$ for all $k=1,2, \ldots q-1, \ell>0$ is the bandwidth parameter, and $k(\bullet)$ is the kernel or lag window. The above estimator of the matrix $V$ was proposed by Lobato, Nankervis and Savin (2002) and was applied to the Box-Pierce test statistic.

As in Lobato et al. the kernel and the bandwidth are assumed to satisfy the following assumptions:

1. The kernel $\mathrm{k}(\bullet)$ belongs to $K$ where $K$ is the class of functions $K=$ $\{k(\bullet): \mathbb{R} ; \longrightarrow[-1,1]\}$ that is symmetric around zero, continuous at zero at all but a finite number of points, and satisfies

$$
k(0)=1, \int_{-\infty}^{\infty}|k(x)| d x<\infty, \int_{-\infty}^{\infty}|\psi(\xi)| d \xi<\infty,
$$

where $\psi(\xi)=(1 / 2 \pi) \int_{-\infty}^{\infty} k(x) e^{i \xi x} d x$. 
2. The bandwidth $\ell$ sequence satisfies

$$
\lim _{T \rightarrow \infty}\left(\frac{1}{\ell}+\frac{\ell}{T}\right)=0
$$

For the consistency of the nonparametric estimator of $\widetilde{C}$, the interested reader is referred to Lobato et al. (2002), lemmas 1 and 2 .

\section{Monte Carlo experiments}

To assess the finite sample performance of alternative versions of the VR statistic, we run simulations and report estimates of the probability of making a type I error. ${ }^{6,7}$ All versions of the $\operatorname{VR}(q)$ test have an asymptotic standard normal distribution. However their finite sample performance differs due to the alternative estimates of the asymptotic variance-covariance matrix $V$. More particularly, we consider the standard VR statistic where the $V$ is an identity matrix, the $\mathrm{VR}^{*}$ under which the $V$ is diagonal, the $\mathrm{VR}_{G P}$ for the general MDS case and two versions of a nonparametric estimate of $V$, the $\widetilde{V}$.

The estimated rejection probabilities are computed for two MDS and two non-MDS examples. The sample sizes considered are 1024 and 5120 observations of the time series $y_{t}$. The aggregation values considered are $q=2,4,8,16,32,64$. The empirical rejection probabilities are compared against nominal $5 \%$ and $10 \%$ levels. The estimates of the empirical rejection probabilities are calculated using 25,000 replications. In the tables, an asterisk denotes that the empirical rejection probability is significantly different at the 0.01 level from the nominal rejection probability where significance is evaluated using a 0.01 level two-sided asymptotic test.

We employ an automatic data-based covariance matrix estimation procedure for the nonparametric estimation of the $\widehat{V}$ matrix. More specifically, the VARHAC procedure suggested by Den Haan and Levin (1997) is applied. This involves estimating a vector autoregression (VAR) of the vector process $\omega_{t}$ where, for each equation in the VAR, the maximum lag order of the elements is selected by the AIC (Akaike, 1973) and the SC (Schwarz, 1978) criteria. The estimated residual series is then recolored to obtain the spectral density matrix at zero frequency of the series $\omega_{t}$. The maximum lag order considered for each sample size and aggregation value is $3 .^{8}$ The same lag length is used for each element of the vector process.

\footnotetext{
${ }^{6}$ The probability of making a type I error is, by definition, the probability of rejecting the null, when it is true.

${ }^{7}$ The random number generator utilized is the modified version of Park and Miller(1998) and uses a period of $4 \times 10^{9}$. The simulations were run in a GNU/Linux environment using the Ox programming language. Calculations were performed on a cluster of Shuttle XPC nodes, with AMD Athlon XP 2800+ (Barton core) processors and connected by a Gigabit ethernet.

${ }^{8}$ Setting the maximum lag order at 3 allows for sufficient degrees of freedom in the estimation of the VARHAC especially for small sample sizes and large aggregation values. It should however be noted that the results of our simulation experiments are not altered by allowing for plausibly larger values of the maximum lag order.
} 
The explicit formula for the VARHAC estimation procedure is given as follows. Denote the vector autoregression (VAR) of the vector process $\omega_{t}=$ $\left[\omega_{1, t}, \omega_{2, t}, \ldots \omega_{K, t}\right]^{\prime}$, where $\omega_{k, t}=\left(y_{t}-\mu\right)\left(y_{t-k}-\mu\right)$ for all $k=1,2, \ldots K$ as $^{9}$ :

$$
\omega_{t}=\Phi_{1} \omega_{t-1}+\Phi_{2} \omega_{t-2}+\ldots+\Phi_{t-S} \omega_{t-S}+\epsilon_{t}
$$

The maximum lag order considered for the VAR is denoted by $S$. First, for each element $k$ of the VAR the AIC and SC are calculated for each lag order $s=1, \ldots, S$. For each element of $\omega_{t}$ the optimal lag order $\widehat{s}$, is chosen as the value that minimizes the model selection criterion.

Then an estimate of $2 \pi$ times the spectral density of the prewhitened residuals is calculated. Let $\bar{S}$ be the largest lag order chosen by the model selection criterion for the $K$ elements of the VAR. The estimated residuals from the restricted vector autoregression are $\widehat{\epsilon}_{t}=\widehat{\omega}_{t}-\sum_{s=1}^{S} \widehat{\Phi}_{s} \widehat{\omega}_{t-s}$ and the innovation covariance matrix is:

$$
\widehat{\Sigma}=\sum_{t=\widehat{S}+1}^{T} \widehat{\epsilon}_{t} \widehat{\epsilon}_{t}^{\prime} / T
$$

where $T$ denotes the sample size of $\omega_{k, t}$.

Finally, using the results of the above steps, the VARHAC estimator of $2 \pi$ times the spectral density at zero frequency of the vector process $\omega_{t}$ is calculated as :

$$
\widehat{C}=\left(I-\sum_{s=1}^{\widehat{S}} \widehat{\Phi}_{s}\right)^{-1} \widehat{\Sigma}\left(I-\sum_{s=1}^{\widehat{S}} \widehat{\Phi}_{s}^{\prime}\right)^{-1}
$$

By the construction of the vector process $\omega_{t}, \widehat{C}$ is an estimate of the variancecovariance matrix $\mathrm{C}$ of the autocorrelation vector $\gamma$ of the scalar process $y_{t}$.

\subsection{MDS examples}

Monte Carlo experiments were conducted using two examples of martingale difference processes. The two MDS models are variants of the GARCH model of Bollerslev (1996). The first is a $\operatorname{GARCH}(1,1)$ model with normal errors while the second is an $\operatorname{EGARCH}(1,1)$ model with normal errors. The $\operatorname{GARCH}(1,1)$ model is $y_{t}=z_{t} \sigma_{t}$ where $z_{t}$ is an i.i.d. sequence and $\sigma_{t}^{2}=\zeta+\alpha y_{t-1}^{2}+\beta \sigma_{t-1}^{2}$, where $\alpha$ and $\beta$ are constants. Provided that the condition $\alpha+\beta<1$ is satisfied, the $y_{t}$ series is covariance stationary. Estimates from stock market data suggest that $\alpha+\beta$ as well as $\beta$ are close to one. ${ }^{10}$ As shown by He and Teräsvirta (1999), the unconditional fourth moment of $y_{t}$ exists for $\operatorname{GARCH}(1,1)$ models if and only if $\beta^{2}+2 \alpha \beta E\left|z_{t}\right|^{2}+\alpha^{2} E\left|z_{t}\right|^{4}<1$. The EGARCH(1,1) model is $y_{t}=z_{t} \sigma_{t}$, where $z_{t}$ is an i.i.d. sequence with zero mean and $\ln \sigma_{t}^{2}=\alpha_{0}+g\left(z_{t-1}\right)+\beta_{0} \ln \sigma_{t-1}^{2}$ where $g\left(z_{t}\right)$ is a well-defined function of $z_{t}$. He, Teräsvirta and Malmsten (2002) show

\footnotetext{
${ }^{9}$ When the $\operatorname{VR}(q)$ test statistic is considered, the dimension of the vector process $\omega_{t}$ is $K=q-1$.

${ }^{10}$ See, for example, Bera and Higgins (1997).
} 
that $y_{t}$ is stationary if $\left|\beta_{0}\right|<1$. Furthermore, He et al. (2002) show that, if $\left\{\mathrm{z}_{t}\right\}$ is Gaussian, then all moments of $y_{t}$ exist.

\subsubsection{Gaussian GARCH $(1,1)$}

$\left\{z_{t}\right\}$ is a sequence of i.i.d. $N(0,1)$ random variables. We set $\zeta=0.001, \alpha=$ 0.09 and $\beta=0.89$. With this parameter setting the He and Teräsvirta (1999) condition for the existence of the fourth moment is satisfied. More precisely for this process, $\gamma(0)=E\left(y_{t}-\mu\right)^{2}=0.05, E\left(y_{t}-\mu\right)^{3} / \gamma(0)^{3 / 2}=0, E\left(y_{t}-\right.$ $\mu)^{4} / \gamma(0)^{2}=5.08$, and $V$ is diagonal. We note that our results are invariant to the value of $\zeta$.

\subsubsection{Gaussian EGARCH $(1,1)$}

$\left\{z_{t}\right\}$ is a sequence of i.i.d. $N(0,1)$ random variables and $\ln \sigma_{t}^{2}=\alpha_{0}+\psi\left|z_{t-1}\right|+$ $\alpha_{0} z_{t-1}+\beta_{0} \ln \sigma_{t-1}^{2}$. We set $\alpha_{0}=0.001, \psi=0.5, \alpha_{0}=-0.2$ and $\beta_{0}=0.95$. We have that (the skewness is an estimate) $\gamma(0)=E\left(y_{t}-\mu\right)^{2}=10.8, E\left(y_{t}-\right.$ $\mu)^{3} / \gamma(0)^{3 / 2}=0, E\left(y_{t}-\mu\right)^{3} / \gamma(0)^{4}=23.4$ and $V$ is non-diagonal.

Table 1 reports the empirical rejection probabilities for the MDS examples for sample sizes of $T=1024$ and 5120 .

(Table 1 around here)

For the GARCH $(1,1)$ process, the diagonal version of the VR test $\left(V^{*}\right)$ performs better relative to the other versions. The rejection probabilities of the $V R_{G P}$ and the two versions of the $\widetilde{V R}$ tests are close to their $V R^{*}$ counterparts, especially for the larger sample size. In particular, for the larger sample the better performance of the $V^{*}$ test is only distinguishable by the fact that the empirical rejection probability is not significantly different from the nominal rejection probability at the $10 \%$ significance level. For the $\operatorname{EGARCH}(1,1)$ process, the $V R_{G P}$ generally performs better than the other tests. However, the $V^{*}$ and the $\widetilde{V}$ work more than satisfactory for small aggregation values $(q)$ and even better for $q=4$.

On the other hand, the use of the identity variance-covariance matrix both for the $\operatorname{GARCH}(1,1)$ and the $\operatorname{EGARCH}(1,1)$ processes results in substantial overrejection of the null. The distortions in the rejection probabilities for the identity covariance matrix become larger as the sample size increases from $T=1024$ to $T=5120$. The main conclusions of the simulation experiments in Table 1 can be summarized as follows : i) for the $\operatorname{GARCH}(1,1)$ and the $\operatorname{EGARCH}(1,1)$ the $V^{*}$ and the $V_{G P}$, respectively, perform better than the other tests, as expected and ii) as the sample size increases to $\mathrm{T}=5120$, the $\widetilde{V}$ versions perform similarly to the diagonal and the Guo-Phillips tests.

\subsection{Non-MDS examples}

In this section we examine the finite sample performance of the tests when the errors are uncorrelated but are non-MDS processes. In recent years, growing evidence that the MDS assumption is too restrictive for financial data has 
emerged as El Babsiri and Zakoian (2001) show. In particular we examine two examples of uncorrelated non-MDS errors. The first is the Granger and Andersen (1978) bilinear model while the second is Tong's (1990) nonlinear moving average model.

\subsubsection{Bilinear model}

The bilinear model is $y_{t}=z_{t}+b z_{t-1} y_{t-2}$ where $\left\{z_{t}\right\}$ is a sequence of i.i.d. $N\left(0, \sigma^{2}\right)$ random variables. It has been fitted to stock return data by Bera and Higgins (1997). The $\mathrm{y}_{t}$ process, although not independent, is uncorrelated and, as long as $b^{2} \sigma^{2}<1$, it is covariance stationary. The condition for the existence of the fourth moment of this process is $3 b^{4} \sigma^{4}<1$. In the experiments we set $\mathrm{b}=0.50$ and $\sigma^{2}=1.0$. For this particular parameter setting we have $\mu=0, \gamma(0)=E\left(y_{t}-\mu\right)^{2}=\sigma^{2} /\left(1-b^{2} \sigma^{2}\right)=1.33, E\left(y_{t}-\mu\right)^{3} / \gamma(0)^{3 / 2}=0$, $E\left(y_{t}-\mu\right)^{4} / \gamma(0)^{2}=3\left(1-b^{4} \sigma^{4}\right) /\left(1-3 b^{4} \sigma^{4}\right)=3.46$ and the $V$ matrix is nondiagonal.

\subsubsection{Nonlinear moving average}

For this model we have $y_{t}=z_{t-1} \cdot z_{t-2} \cdot\left(z_{t-2}+z_{t}+c\right)$ where $\left\{z_{t}\right\}$ is a sequence of i.i.d. $N(0,1)$ random variables and $c=1.0$. For this process the second, third and fourth moments are $E\left(y_{t}-\mu\right)^{2}=5, E\left(y_{t}-\mu\right)^{3} / \gamma(0)^{3 / 2}=0$ and $E\left(y_{t}-\mu\right)^{4} / \gamma(0)^{4}=37.80$. The asymptotic variance-covariance matrix $V$ is non-diagonal for the nonlinear moving average model.

The results of the Monte Carlo experiments, when the underlying DGPs of the $y_{t}$ series are non-MDS processes, are presented in Table 2.

\section{(Table 2 around here)}

For $T=1024$, the two versions of the $\widetilde{V}$, work relatively better than the other tests for aggregation values up to $q=16$. In particular, both for the bilinear and the non linear moving average process, the $\widetilde{V}$ considerably outperforms the alternative versions. The $\widetilde{V}$ tests perform better at the lager sample size for both processes. The difference between the nominal and empirical rejection probabilities is around 1\% for both examples and for all values of $q$ at $\mathrm{T}=5120$. The results of Table 2 are in line with our a priori expectation that the $\widetilde{V R}$ tests should perform better for the non-MDS examples since only these tests use consistent estimators of $V$.

\section{Power}

We report the empirical power of the alternative versions of the VR test where the DGPs of the $\mathrm{y}_{t}$ series are either AR or MA processes with uncorrelated errors. ${ }^{11}$ The errors $\left(u_{t}\right)$ for both the AR and the MA specifications, are assumed

\footnotetext{
${ }^{11}$ Since $y_{t}$ is defined as the first difference of the $x_{t}$ series, when the fomer follows an AR or an MA process, $x_{t}$ follows an $\operatorname{ARIMA}(1,1,0)$ or an $\operatorname{ARIMA}(0,1,1)$, respectively.
} 
to be generated by the processes examined in the previous section and hence are uncorrelated. ${ }^{12}$

For the AR model we consider the $\mathrm{AR}(1)$ and the $\mathrm{AR}(6)$ specifications. The $\mathrm{AR}(1)$ model is $y_{t}=\phi y_{t-1}+u_{t}$ while the $\mathrm{AR}(6)$ model being considered is $y_{t}=\phi \sum_{j=1}^{6} \frac{7-j}{6} y_{t-j}+u_{t}$. The $y_{t}$ series following an $\operatorname{AR}(p)$ model is covariance stationary as long as the roots of $1-\phi_{1} z-\phi_{1} z^{2}-\ldots-\phi_{p} z^{p}=0$ all lie outside the unit circle. The parameter values of $\phi$ are chosen to range from 0.075 to 0.200 , for the case of the $\operatorname{AR}(1)$, while for the $\operatorname{AR}(6)$ model the values of $\phi$ range from 0.05 to $0.1 .^{13}$ It follows from the definition of the AR models and the uncorrelatedness of the errors that a zero coefficient for $\phi$ yields an uncorrelated $y_{t}$ series.

We also allow the $y_{t}$ series to be either an MA(1) or an MA(2) model. The MA(1) model is $y_{t}=u_{t}+\theta_{0} u_{t-1}$ while the MA(2) model is $y_{t}=u_{t}+\theta_{1} u_{t-1}+$ $\theta_{2} u_{t-2}$. However, unless the coefficient $\theta_{0}$, for the $\mathrm{MA}(1)$, and $\theta_{1}, \theta_{2}$ for the MA(2) process are different from zero, the $y_{t}$ process is no longer uncorrelated. The MA processes are covariance stationary no matter what value the moving average coefficients $\theta_{i}$ might take. However any MA(q) can be written as an $\operatorname{AR}(\infty)$ process as long as the roots of the polynomial $\left(1+\theta_{1} L+\theta_{2} L^{2}+\ldots+\right.$ $\left.+\theta_{q} L^{q}\right)=0$ lie outside the unit circle. For the MA(1) case we set the value of $\theta_{0}$ so as to have an invertible MA and the first autocorrelation $(\rho(1))$ to take values in the range of 0.075 to 0.150 . For the MA(2) case we find the appropriate $\theta_{1}$ and $\theta_{2}$, by setting $\rho(1)=\rho(2)=\bar{\rho}$ and the values of $\bar{\rho}$ range from 0.040 to 0.085 . Again, the appropriate values of $\theta_{1}$ and $\theta_{2}$ are selected in order to have an invertible $\mathrm{MA}(2)$ process. In the power comparisons, both for the $\mathrm{AR}$ and the MA models, the empirical powers are adjusted so that the empirical rejections under the null are exactly $10 \%$ and $5 \%$ for two-sided tests.

Table 3 reports the empirical power when the underlying DGP is an AR (1) model with uncorrelated errors. ${ }^{14}$

(Table 3 around here)

In Panel A and B of this table we report the empirical power of the VR-tests for aggregation values of $q=4$ and $q=8$, respectively. For the $\operatorname{AR}(1)$ model the $\widetilde{V R}(A I C)$ and $\widetilde{V R}(S C)$ outperform the other VR alternatives. In particular when the errors follow either an $\operatorname{EGARCH}(1,1)$, bilinear or non-linear moving average process the increase in power can be up to 10 percentage points compared to the $\mathrm{VR}^{*}$ and the $\mathrm{VR}_{G P}$ versions. For example, in panel $\mathrm{B}$, at the

\footnotetext{
${ }^{12}$ In the power comparisons, caution in the selection of the appropriate $q$ value for a given sample size should be exercised due to the variance ratio test's skewed empirical distribution. Lo and MacKinlay (1989) select values of $q$ so that the maximum value of the ratio $q / T$ is 0.5 .

${ }^{13}$ In addition to selecting appropriate parameter values for $\phi$ to ensure stationarity, the AR models are simulated with an approximate stationary startup. This involves taking the last $T$ random observations from a simulated sequence of $T+500$ where the startup values are set equal to zero.

${ }^{14}$ The power of a test statistic (or 1 minus the probability of making a Type II error) is by definition the probability of rejecting the null hypothesis when it is actually false.
} 
$10 \%$ level when the errors follow an $\operatorname{EGARCH}(1,1)$ and $\phi=0.200$ the empirical rejection probabilities for the $\mathrm{VR}^{*}, \mathrm{VR}_{G P}$ and $\widetilde{V R}$ are $60.6 \%, 64.3 \%$ and $72.7 \%$, respectively.

The empirical rejection probabilities for the $\mathrm{AR}(6)$ process are provided in Table 4 .

(Table 4 around here)

For the $\operatorname{AR}(6)$ model with $\operatorname{GARCH}(1,1)$ errors, the diagonal, $\mathrm{VR}_{G P}$ and the $\widetilde{V}$ versions of the VR-test seem to have similar power. When the errors follow an $\operatorname{EGARCH}(1,1)$ process the $\mathrm{VR}_{G P}$ performs better than the $\mathrm{VR}^{*}$ by $2-5$ percentage points while the two $\widetilde{V R}$ versions result in a gain of power of 313 percentage points relative to the $\mathrm{VR}^{*}$. Considering bilinear and nonlinear moving average errors does not change the fact that the $\mathrm{VR}_{G P}$ and the $\overline{V R}$ perform better than the $\mathrm{VR}^{*}$ version. It should be noted that for the $\operatorname{AR}(6)$ with bilinear errors the $\mathrm{VR}_{G P}$ performs slightly better than the $\widetilde{V R}$ and vice versa for the $\mathrm{AR}(6)$ with nonlinear moving average errors.

The empirical rejection probabilities when $y_{t}$ is an $\mathrm{MA}(1)$ process are reported in Table 5 .

(Table 5 around here)

The main message from Table 5 is that the non-diagonal versions of the VR test have more power and this is in line with the findings for the AR models. In particular, the use of the VARHAC covariance matrix results in more powerful tests. The resultant increase in power can be up to 10 percentage points compared to the diagonal variance-covariance matrix and up to 8 percentage points than the $\mathrm{VR}_{G P}$ versions of the test.

The simulation outcomes for the MA(2) process are presented in Table 6 .

(Table 6 around here)

The results provide further evidence that the $\widetilde{V R}$ and $\mathrm{VR}_{G P}$ have more power than the standard identity and diagonal versions of the VR test statistic. Once again the $\widetilde{V R}$ performs better than the $\mathrm{VR}_{G P}$ and the improvement is more evident for $\operatorname{EGARCH}(1,1)$ errors.

\section{Conclusions}

We develop new extensions of the variance ratio test statistic to test for uncorrelatedness in the presence of statistical dependence that characterises many financial and economic time series. These extensions are similar in spirit to the extension of the Box-Pierce suggested by Lobato,Nankervis and Savin (2002). They employ consistent estimates of the asymptotic variance-covariance matrix of the sample autocorrelations of the increments $y_{t}$. Lo and MacKinlay (1988, 1989) show that when the increments are either iid or are conditionally heteroskedastic, the asymptotic variance-covariance matrix of the autocorrelations 
is either an identity or diagonal matrix, respectively. However in more general cases, this matrix is no longer diagonal in cases where the sample autocorrelations of $y_{t}$ are not asymptotically uncorrelated. For example, the asymptotic covariance matrix of the sample autocorrelations is non-diagonal when the underlying DGP of the $y_{t}$ is either a GARCH process with non-symmetric errors or an EGARCH process.

The finite sample performance of the suggested extensions of the VR test is evaluated in a Monte Carlo study. The size and power properties of the alternative VR versions are studied both when the underlying DGPs are martingale difference sequences (MDS) or non-MDS processes. The MDS employed for size evaluation are the $\operatorname{GARCH}(1,1)$ and $\operatorname{EGARCH}(1,1)$ with normal errors while the non-MDS examples are a bilinear and non-linear moving average model. The alternative hypothesis for the power comparison is that the underlying process for the increments is either an $\mathrm{AR}(1), \mathrm{AR}(6), \mathrm{MA}(1)$ or $\mathrm{MA}(2)$ model.

In the MDS framework, the $\mathrm{VR}^{*}$ (diagonal case) version is best sized for the GARCH $(1,1)$ model as expected whereas the $\mathrm{VR}_{G P}$ version performs better for the $\operatorname{EGARCH}(1,1)$ model. The empirical rejections for both versions of our proposed $V R$ test are very close to their nominal levels for the larger sample size. More interestingly, the results of the power study using adjusted critical values show that the non-diagonal versions of the VR test are more powerful. The use of our proposed $\widetilde{V R}(\mathrm{AIC})$ and $\widetilde{V R}(\mathrm{SC})$ tests result in an increase in power of up to 10 percentage points. 


\section{References}

[1] Akaike, H. (1973) Information theory and the extension of the maximum likelihood principle. In B. Petrov \& F. Csaki (eds.), Second International Symposium on Information Theory, 267-281. Budapest: Akailseoniai-Kiudo

[2] Bera, A.K. \& M.L. Higgins (1997) ARCH and bilinearity as competing models for nonlinear dependence. Journal of Business and Economic Statistics, Supplement 8, 27-41, 85-97

[3] Black, F. (1976) Studies of stock market volatility changes. Proceedings of the American Statistical Association, Business and Economic Studies Section, $177-181$

[4] Bollerslev, T. (1986) Generalized autoregressive conditional heteroskedasticity. Journal of Econometrics 31,307-327

[5] Chen, W.W. \& R.S. Deo (2004) The variance ratio statistic at large horizons. Forthcoming Econometric Theory

[6] Den Haan, W.J. \& A. Levin (1997) A practisoner's guide to robust covariance matrix estimation. In G.S. Maddala \& C.R. Rao (eds.), Handbook of Statistics: Robust Inference, vol. 15, 291-341. Amsterdam: North-Holland.

[7] Ding, Z., C.W.J. Granger \& R.F. Engle (1993) A long memory property of stock market returns and a new model. Journal of Empirical Finance, 1, 83-106

[8] El Babsiri,M and J.-M. Zakoian (2001) Contemporaneous asymmetry in GARCH processes. Journal of Econometrics 101, 257-294.

[9] Fernández, C. \& M.Steel (1998) On Bayesian modelling of fat tails and skewness. Journal of the American Statistical Association, 93, 359-371

[10] Granger, C.W.J. \& A.P. Andersen (1978) An Introduction to Bilinear Time Series Models. Gottingen: Vanenhoek and Ruprecht

[11] Guo, B.B. \& P.C.B. Phillips (1998) Testing for autocorrelation and unit roots in the presence of conditional heteroskedasticity of unknown form. Cowles Foundation for Research in Economics, Yale University.

[12] Hansen B. (1994) Autoregressive conditional density estimation. International Economic Review. 35, 705-730

[13] He, C. \& T.Teräsvirta (1999) Properties of moments of a family of GARCH processes. Journal of Econometrics 92, 173-192

[14] He, C. , T.Teräsvirta \& H. Malmsten (2002) Moment structure of a family of first-order exponential GARCH models. Econometric Theory 18, 868885 . 
[15] Lo, A.W. \& A.C. MacKinlay (1988) Stock market prices do not follow random walks: Evidence from a simple specification test. Review of Financial Studies 1, 41-66.

[16] Lo, A.W. \& A.C. MacKinlay (1989) The size and power of the variance ratio test in finite samples: A Monte Carlo investigation. Journal of Econometrics 40, 203-238.

[17] Lobato, I.N., J.C. Nankervis \& N.E. Savin (2002) Testing for zero autocorrelation in the presence of statistical dependence. Econometric Theory 18, 730-743.

[18] Lambert, P. \& S. Laurent (2001) Modelling financial time series using GARCH-type models and a skewed Student density. Mimeo, Université de Liège.

[19] Nelson, D. (1991) Conditional heteroskedasticity in asset returns: a new approach. Econometrica 59, 349-370

[20] Park, S. \& Miller, K.(1998) Random number generators: Good ones are hard to find. Communications of the ACM, 31, 1192-1201.

[21] Richardson, M \& J. Stock (1989) Drawing Inferences from Statistics based on Multi-Year Asset Returns. Journal of Financial Economics 25, 323-348.

[22] Schwarz, G. (1978) Estimating the dimensions of a model. Annals of Statistics 33, 300-308.

[23] Tong H. (1990) Nonlinear Time series. Oxford: Oxford University Press.

[24] Zakoian, J.-M. (1994) Threshold heteroskedasticity models. Journal of Economic Dynamics and Control. 15, 931-955 
Table 1. Rejection probabilities (\%) of tests: MDS examples

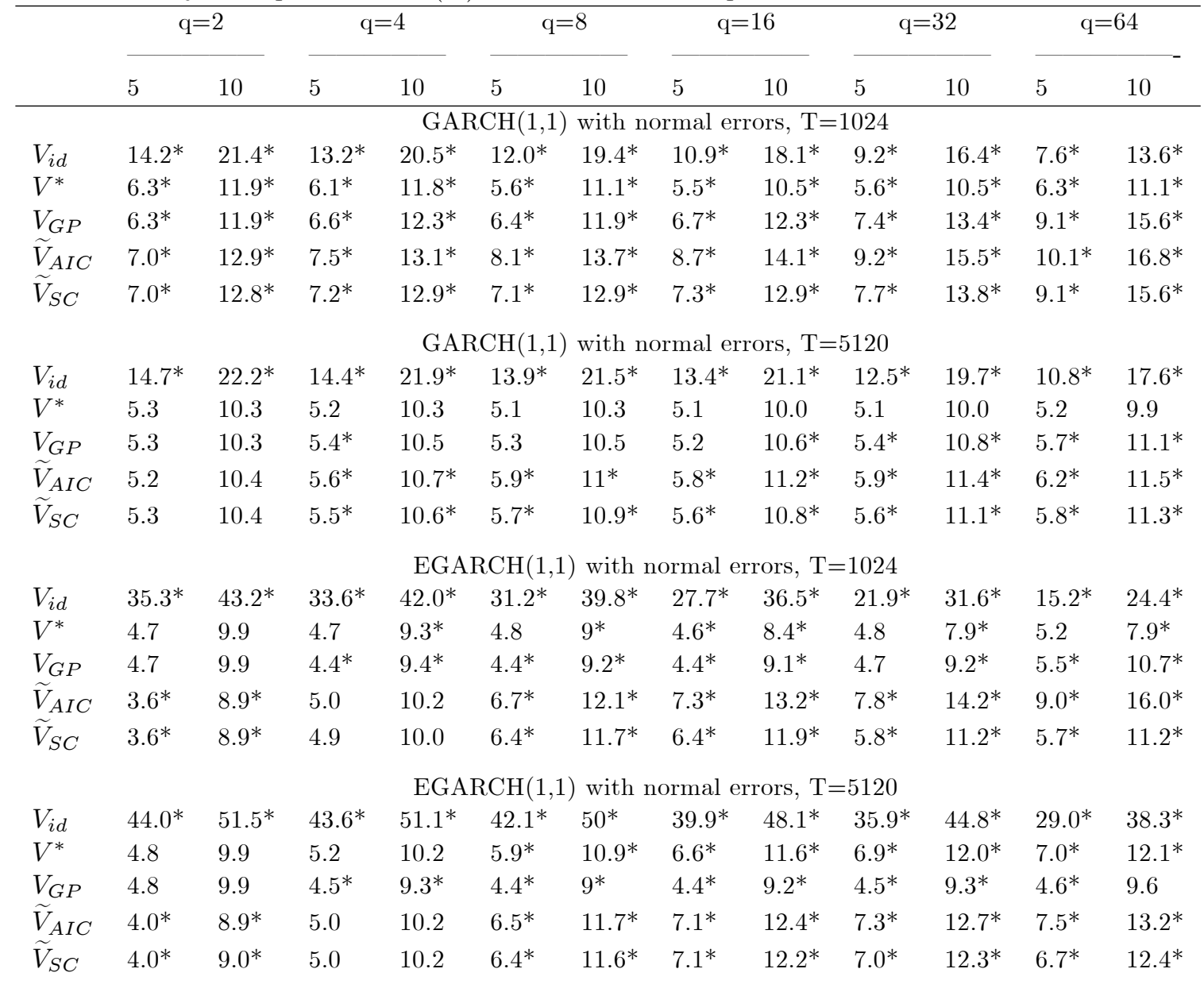

Notes: The number of replications is 25,000. An asterisk denotes that the empirical rejection probability is significantly different at the 0.01 level from the nominal rejection probability, where the significance is evaluated using a 0.01 level two-sided asymptotic test. 
Table 2. Rejection probabilities (\%) of tests: Non-MDS examples

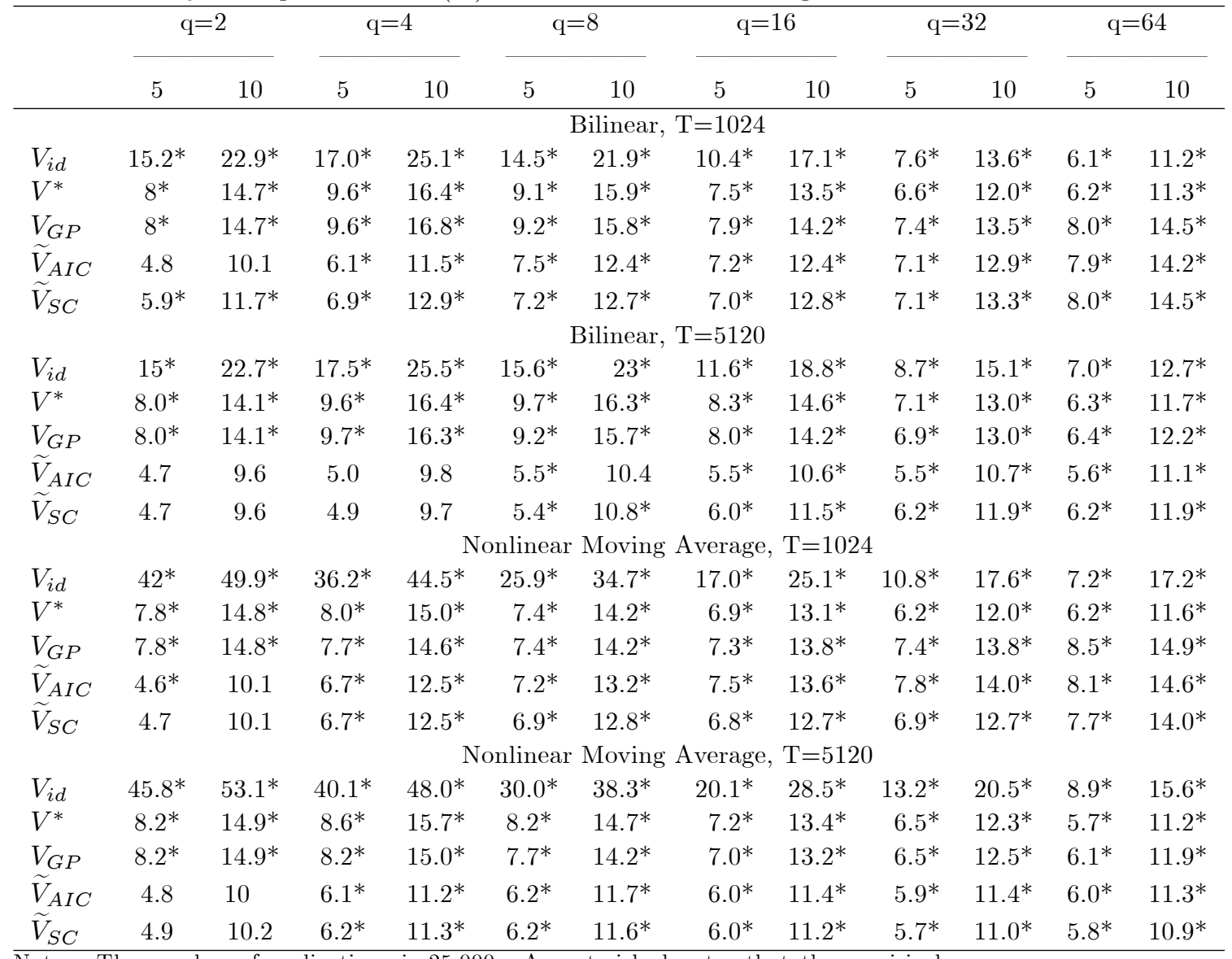

Notes: The number of replications is 25,000 . An asterisk denotes that the empirical rejection probability is significantly different at the 0.01 level from the nominal rejection probability, where the significance is evaluated using a 0.01 level two-sided asymptotic test. 
Table 3A. Empirical power for an AR(1) process

\begin{tabular}{|c|c|c|c|c|c|c|c|c|c|c|}
\hline \multirow{3}{*}{$\phi$} & \multicolumn{10}{|c|}{ Panel A: $q=4$} \\
\hline & \multicolumn{2}{|c|}{0.075} & \multicolumn{2}{|c|}{0.100} & \multicolumn{2}{|c|}{0.125} & \multicolumn{2}{|c|}{0.150} & \multicolumn{2}{|c|}{0.200} \\
\hline & 10 & 5 & 10 & 5 & 10 & 5 & 10 & 5 & 10 & 5 \\
\hline & \multicolumn{10}{|c|}{ GARCH $(1,1)$ with normal errors } \\
\hline$V_{i d}$ & 50.3 & 36.9 & 70.7 & 57.7 & 86.1 & 77.1 & 94.6 & 89.9 & 99.6 & 99.0 \\
\hline$V^{*}$ & 51.0 & 38.3 & 71.1 & 59.0 & 85.8 & 77.7 & 94.2 & 89.7 & 99.4 & 98.6 \\
\hline$V_{G P}$ & 51.7 & 39.3 & 71.7 & 60.1 & 86.3 & 78.5 & 94.4 & 90.3 & 99.4 & 98.7 \\
\hline$\widetilde{V}_{A I C}$ & 54.5 & 42.4 & 74.8 & 63.8 & 88.4 & 81.7 & 95.5 & 92.1 & 99.6 & 99.1 \\
\hline \multirow[t]{2}{*}{$\widetilde{V}_{S C}$} & 53.7 & 41.3 & 73.9 & 62.6 & 87.8 & 80.7 & 95.2 & 91.5 & 99.5 & 98.9 \\
\hline & \multicolumn{10}{|c|}{$\operatorname{EGARCH}(1,1)$ with normal errors } \\
\hline$V_{i d}$ & 18.5 & 9.4 & 27.0 & 14.3 & 38.2 & 21.7 & 25.6 & 14.3 & 76.5 & 58.1 \\
\hline$V^{*}$ & 22.7 & 14.1 & 33.7 & 22.3 & 46.3 & 33.5 & 30.8 & 20.1 & 80.1 & 70.4 \\
\hline$V_{G P}$ & 24.3 & 15.2 & 36.0 & 24.6 & 48.8 & 36.7 & 32.5 & 21.7 & 82.1 & 73.5 \\
\hline$\widetilde{V}_{A I C}$ & 28.3 & 19.4 & 41.3 & 31.1 & 55.6 & 44.5 & 35.6 & 24.3 & 86.9 & 80.3 \\
\hline \multirow[t]{2}{*}{$\widetilde{V}_{S C}$} & 28.4 & 19.5 & 41.4 & 31.0 & 55.8 & 44.5 & 35.1 & 23.9 & 87.1 & 80.4 \\
\hline & \multicolumn{10}{|c|}{ Bilinear } \\
\hline$V_{i d}$ & 37.0 & 25.0 & 55.1 & 41.1 & 72.6 & 59.7 & 85.7 & 76.2 & 97.9 & 95.3 \\
\hline$V^{*}$ & 37.5 & 26.2 & 55.6 & 42.9 & 72.8 & 61.5 & 85.9 & 77.8 & 97.9 & 95.8 \\
\hline$V_{G P}$ & 38.4 & 27.6 & 56.8 & 44.9 & 74.1 & 63.8 & 86.8 & 79.8 & 98.1 & 96.3 \\
\hline$\widetilde{V}_{A I C}$ & 43.5 & 31.6 & 63.4 & 50.8 & 80.1 & 70.3 & 91.1 & 85.1 & 99.2 & 97.9 \\
\hline \multirow[t]{2}{*}{$\widetilde{V}_{S C}$} & 42.1 & 30.1 & 61.6 & 49.0 & 78.5 & 68.2 & 89.9 & 83.4 & 98.9 & 97.4 \\
\hline & \multicolumn{10}{|c|}{ Nonlinear Moving Average } \\
\hline$V_{i d}$ & 19.4 & 10.5 & 28.1 & 16.1 & 39.3 & 24.2 & 52.1 & 34.9 & 77.0 & 61.4 \\
\hline$V^{*}$ & 24.8 & 15.8 & 36.2 & 25.2 & 49.2 & 36.8 & 62.2 & 50.3 & 83.4 & 75.0 \\
\hline$V_{G P}$ & 27.0 & 17.8 & 39.3 & 28.6 & 53.1 & 41.6 & 66.3 & 55.7 & 86.0 & 79.6 \\
\hline$\widetilde{V}_{A I C}$ & 30.0 & 20.4 & 43.6 & 32.4 & 58.2 & 46.7 & 71.7 & 61.0 & 89.7 & 83.6 \\
\hline$\widetilde{V}_{S C}$ & 30.0 & 20.2 & 43.7 & 32.2 & 58.4 & 46.3 & 71.7 & 60.9 & 89.7 & 83.3 \\
\hline
\end{tabular}

Notes: The number of replications is 25,000. The critical values are adjusted so that empirical rejection probabilities of the tests under the null are exactly 0.1 and 0.05 for twosided tests. The empirical powers are in percentages. 
Table 3B. Empirical power for an AR(1) process

\begin{tabular}{|c|c|c|c|c|c|c|c|c|c|c|}
\hline \multirow{3}{*}{$\phi$} & \multicolumn{10}{|c|}{ Panel B: $q=8$} \\
\hline & \multicolumn{2}{|c|}{0.075} & \multicolumn{2}{|c|}{0.100} & \multicolumn{2}{|c|}{0.125} & \multicolumn{2}{|c|}{0.150} & \multicolumn{2}{|c|}{0.200} \\
\hline & 10 & 5 & 10 & 5 & 10 & 5 & 10 & 5 & 10 & 5 \\
\hline & \multicolumn{10}{|c|}{ GARCH $(1,1)$ with normal errors } \\
\hline$V_{i d}$ & 34.0 & 22.1 & 49.0 & 35.4 & 64.8 & 50.8 & 78.2 & 66.5 & 94.4 & 89.4 \\
\hline$V^{*}$ & 34.6 & 23.5 & 49.9 & 36.9 & 65.1 & 52.6 & 78.2 & 67.7 & 93.9 & 89.2 \\
\hline$V_{G P}$ & 35.4 & 24.6 & 51.0 & 38.2 & 66.2 & 54.1 & 79.1 & 69.0 & 94.3 & 90.1 \\
\hline$\widetilde{V}_{A I C}$ & 38.3 & 26.9 & 54.7 & 41.9 & 70.1 & 58.5 & 82.2 & 73.2 & 95.8 & 92.0 \\
\hline \multirow[t]{2}{*}{$\widetilde{V}_{S C}$} & 36.4 & 25.6 & 52.5 & 39.6 & 67.5 & 55.7 & 80.2 & 70.1 & 94.8 & 90.2 \\
\hline & \multicolumn{10}{|c|}{$\operatorname{EGARCH}(1,1)$ with normal errors } \\
\hline$V_{i d}$ & 14.2 & 6.7 & 18.8 & 9.2 & 25.0 & 12.6 & 33.1 & 17.6 & 53.2 & 32.1 \\
\hline$V^{*}$ & 16.9 & 9.1 & 22.6 & 13.2 & 30.4 & 18.8 & 40.0 & 26.1 & 60.6 & 45.4 \\
\hline$V_{G P}$ & 17.9 & 10.6 & 24.7 & 15.7 & 33.2 & 22.5 & 43.3 & 31.2 & 64.3 & 52.0 \\
\hline$\widetilde{V}_{A I C}$ & 21.2 & 12.9 & 30.0 & 19.7 & 40.2 & 28.8 & 52.0 & 39.3 & 72.7 & 62.0 \\
\hline \multirow[t]{2}{*}{$\widetilde{V}_{S C}$} & 21.2 & 12.9 & 29.9 & 19.6 & 40.2 & 28.7 & 51.5 & 39.3 & 72.7 & 61.9 \\
\hline & \multicolumn{10}{|c|}{ Bilinear } \\
\hline$V_{i d}$ & 26.7 & 16.4 & 39.1 & 26.5 & 53.5 & 39.2 & 67.6 & 53.9 & 88.9 & 80.5 \\
\hline$V^{*}$ & 26.7 & 17.1 & 39.7 & 27.3 & 54.1 & 40.7 & 68.0 & 55.3 & 89.0 & 81.5 \\
\hline$V_{G P}$ & 28.1 & 18.0 & 41.1 & 29.0 & 55.7 & 42.9 & 69.8 & 57.6 & 90.1 & 83.1 \\
\hline$V_{A I C}$ & 32.6 & 21.9 & 47.5 & 35.3 & 63.0 & 51.3 & 77.0 & 66.7 & 94.0 & 89.2 \\
\hline \multirow[t]{2}{*}{$V_{S C}$} & 30.2 & 19.4 & 44.0 & 31.3 & 58.7 & 45.3 & 72.8 & 59.9 & 91.4 & 84.3 \\
\hline & \multicolumn{10}{|c|}{ Nonlinear Moving Average } \\
\hline$V_{i d}$ & 17.5 & 9.7 & 24.4 & 14.4 & 34.1 & 21.0 & 45.7 & 30.2 & 69.5 & 53.9 \\
\hline$V^{*}$ & 20.9 & 12.6 & 29.8 & 19.8 & 40.8 & 28.9 & 52.8 & 40.2 & 75.2 & 64.2 \\
\hline$V_{G P}$ & 23.0 & 14.8 & 32.9 & 23.3 & 44.9 & 34.0 & 56.9 & 46.2 & 78.7 & 70.1 \\
\hline$\widetilde{V}_{A I C}$ & 25.4 & 16.5 & 36.8 & 26.2 & 49.2 & 37.8 & 62.0 & 50.7 & 83.0 & 74.4 \\
\hline$\widetilde{V}_{S C}$ & 25.0 & 16.9 & 36.4 & 26.7 & 48.9 & 38.5 & 61.4 & 51.3 & 82.7 & 75.0 \\
\hline
\end{tabular}

Notes: The number of replications is 25,000 and $\mathrm{T}=1024$. The critical values are adjusted so that empirical rejection probabilities of the tests under the null are exactly 0.1 and 0.05 for two-sided tests. The empirical powers are in percentages. 
Table 4. Empirical power for an $\mathrm{AR}(6)$ process

\begin{tabular}{|c|c|c|c|c|c|c|c|c|c|c|c|c|}
\hline \multirow{3}{*}{$\phi$} & \multicolumn{6}{|c|}{$\mathrm{q}=4$} & \multicolumn{6}{|c|}{$q=8$} \\
\hline & \multicolumn{2}{|c|}{0.050} & \multicolumn{2}{|c|}{0.075} & \multicolumn{2}{|c|}{0.100} & \multicolumn{2}{|c|}{0.050} & \multicolumn{2}{|c|}{0.075} & \multicolumn{2}{|c|}{0.100} \\
\hline & 10 & 5 & 10 & 5 & 10 & 5 & 10 & 5 & 10 & 5 & 10 & 5 \\
\hline & \multicolumn{12}{|c|}{$\operatorname{GARCH}(1,1)$ with normal errors } \\
\hline$V_{i d}$ & 65.6 & 52.8 & 92.2 & 86.7 & 99.2 & 98.3 & 72.1 & 60.0 & 95.6 & 91.9 & 99.7 & 99.4 \\
\hline$V^{*}$ & 66.3 & 54.3 & 91.9 & 86.7 & 99.0 & 98.0 & 72.4 & 61.4 & 95.3 & 91.8 & 99.6 & 99.1 \\
\hline$V_{G P}$ & 67.4 & 56.1 & 92.6 & 87.9 & 99.1 & 98.3 & 74.2 & 63.7 & 95.8 & 92.9 & 99.6 & 99.3 \\
\hline$\widetilde{V}_{A I C}$ & 66.5 & 54.8 & 92.0 & 86.3 & 98.9 & 97.7 & 75.9 & 65.4 & 96.3 & 93.0 & 99.6 & 99.2 \\
\hline$\widetilde{V}_{S C}$ & 67.3 & 55.2 & 92.2 & 86.6 & 98.9 & 97.7 & 75.0 & 64.9 & 96.0 & 92.7 & 99.6 & 99.0 \\
\hline & \multicolumn{12}{|c|}{$\operatorname{EGARCH}(1,1)$ with normal errors } \\
\hline$V_{i d}$ & 25.8 & 14.1 & 48.4 & 31.2 & 74.0 & 57.5 & 29.8 & 16.0 & 58.2 & 37.7 & 84.4 & 68.4 \\
\hline$V^{*}$ & 31.6 & 21.1 & 56.0 & 44.0 & 78.3 & 69.3 & 36.0 & 23.6 & 64.5 & 50.8 & 86.4 & 77.6 \\
\hline$V_{G P}$ & 33.9 & 23.5 & 59.1 & 47.9 & 80.9 & 73.0 & 39.9 & 28.7 & 69.0 & 58.4 & 89.2 & 83.3 \\
\hline$\widetilde{V}_{A I C}$ & 34.9 & 25.1 & 60.3 & 49.2 & 81.3 & 72.6 & 45.4 & 33.3 & 74.4 & 63.8 & 91.5 & 85.3 \\
\hline$\widetilde{V}_{S C}$ & 35.0 & 25.3 & 60.6 & 49.3 & 81.4 & 72.8 & 45.5 & 33.5 & 74.5 & 64.1 & 91.5 & 85.4 \\
\hline & \multicolumn{12}{|c|}{ Bilinear } \\
\hline$V_{i d}$ & 51.0 & 38.0 & 82.2 & 72.7 & 96.9 & 94.0 & 61.6 & 47.9 & 91.1 & 84.3 & 99.3 & 98.4 \\
\hline$V^{*}$ & 51.2 & 39.2 & 82.4 & 73.7 & 96.9 & 94.2 & 61.9 & 49.3 & 91.3 & 85.2 & 99.3 & 98.4 \\
\hline$V_{G P}$ & 53.3 & 42.2 & 84.2 & 77.0 & 97.4 & 95.5 & 65.3 & 53.6 & 93.1 & 88.3 & 99.6 & 99.0 \\
\hline$\widetilde{V}_{A I C}$ & 55.1 & 42.4 & 85.3 & 76.8 & 97.7 & 95.0 & 70.1 & 58.7 & 94.8 & 90.8 & 99.7 & 99.2 \\
\hline$\tilde{V}_{S C}$ & 54.0 & 41.4 & 84.5 & 75.5 & 97.2 & 94.2 & 68.1 & 56.7 & 93.9 & 89.4 & 99.5 & 98.7 \\
\hline \multicolumn{13}{|c|}{ Nonlinear Moving Average } \\
\hline$V_{i d}$ & 27.2 & 16.0 & 50.4 & 34.8 & 75.9 & 61.8 & 40.9 & 27.5 & 73.3 & 59.8 & 93.3 & 87.7 \\
\hline$V^{*}$ & 36.0 & 26.1 & 62.4 & 52.1 & 83.8 & 77.6 & 50.4 & 39.4 & 80.2 & 72.6 & 94.9 & 91.9 \\
\hline$V_{G P}$ & 39.1 & 29.5 & 66.0 & 57.2 & 86.2 & 81.1 & 54.5 & 45.5 & 83.2 & 77.4 & 96.0 & 93.9 \\
\hline$\widetilde{\widetilde{V}}_{A I C}$ & 37.9 & 27.2 & 64.4 & 53.3 & 85.1 & 77.1 & 54.0 & 43.1 & 82.6 & 74.4 & 95.6 & 91.9 \\
\hline$\widetilde{V}_{S C}$ & 38.6 & 27.5 & 64.8 & 53.5 & 85.3 & 77.2 & 55.3 & 45.7 & 83.1 & 76.3 & 95.7 & 92.5 \\
\hline
\end{tabular}

Notes: The number of replications is 25,000 and $T=1024$. The critical values are adjusted so that empirical rejection probabilities of the tests under the null are exactly 0.1 and 0.05 for two-sided tests. The empirical powers are in percentages. 
Table 5. Empirical power for an MA(1) process

\begin{tabular}{|c|c|c|c|c|c|c|c|c|c|c|c|c|c|c|c|c|}
\hline \multirow{3}{*}{$\rho_{1}$} & \multicolumn{8}{|c|}{$q=4$} & \multicolumn{8}{|c|}{$q=8$} \\
\hline & \multicolumn{2}{|c|}{0.075} & \multicolumn{2}{|c|}{0.100} & \multicolumn{2}{|c|}{0.125} & \multicolumn{2}{|c|}{0.150} & \multicolumn{2}{|c|}{0.075} & \multicolumn{2}{|c|}{0.100} & \multicolumn{2}{|c|}{0.125} & \multicolumn{2}{|c|}{0.150} \\
\hline & 10 & 5 & 10 & 5 & 10 & 5 & 10 & 5 & 10 & 5 & 10 & 5 & 10 & 5 & 10 & 5 \\
\hline & \multicolumn{16}{|c|}{$\operatorname{GARCH}(1,1)$ with normal errors } \\
\hline$V_{i d}$ & 46.8 & 33.7 & 65.2 & 51.6 & 80.4 & 69.5 & 90.3 & 83.3 & 31.2 & 20.0 & 43.5 & 30.5 & 56.4 & 42.5 & 68.2 & 55.0 \\
\hline$V^{*}$ & 46.5 & 34.1 & 64.9 & 52.0 & 79.8 & 69.5 & 89.4 & 82.5 & 31.3 & 20.7 & 43.5 & 31.4 & 56.5 & 43.3 & 67.8 & 55.8 \\
\hline$V_{G P}$ & 46.9 & 34.8 & 65.5 & 53.0 & 80.2 & 70.3 & 89.7 & 83.2 & 31.9 & 21.6 & 44.4 & 32.4 & 57.5 & 44.7 & 68.7 & 57.4 \\
\hline$\widetilde{V}_{A I C}$ & 49.9 & 38.0 & 69.0 & 57.1 & 83.0 & 74.3 & 91.7 & 86.1 & 34.6 & 23.6 & 48.3 & 35.7 & 61.3 & 49.1 & 72.6 & 61.7 \\
\hline$\widetilde{V}_{S C}$ & \multicolumn{12}{|c|}{ EGARCH $(1,1)$ with normal errors } & 58.8 & 46.1 & 70.0 & 58.3 \\
\hline$V_{i d}$ & 17.9 & 9.2 & 24.9 & 13.2 & 33.9 & 18.9 & 44.5 & 26.3 & 13.6 & 6.5 & 17.2 & 8.3 & 22.2 & 10.9 & 27.7 & 14.1 \\
\hline$V^{*}$ & 21.5 & 13.2 & 30.6 & 19.8 & 41.1 & 28.6 & 51.7 & 39.0 & 15.6 & 8.3 & 20.5 & 11.5 & 26.1 & 15.6 & 33.0 & 20.7 \\
\hline$V_{G P}$ & 23.0 & 14.3 & 32.7 & 21.9 & 43.5 & 31.6 & 54.5 & 42.0 & 16.7 & 9.9 & 22.3 & 13.9 & 28.8 & 18.9 & 36.2 & 24.7 \\
\hline$\widetilde{V}_{A I C}$ & 26.8 & 18.0 & 38.0 & 27.8 & 50.2 & 39.4 & 62.0 & 51.1 & 19.9 & 11.8 & 27.1 & 17.4 & 35.3 & 24.3 & 44.1 & 32.1 \\
\hline$\widetilde{V}_{S C}$ & \multicolumn{15}{|c|}{ Bilinear } & 31.9 \\
\hline$V_{i d}$ & 34.7 & 22.9 & 50.7 & 36.8 & 66.3 & 52.7 & 79.5 & 68.0 & 24.6 & 14.9 & 34.7 & 22.9 & 46.0 & 32.6 & 58.0 & 43.5 \\
\hline$V^{*}$ & 35.0 & 24.1 & 50.9 & 38.5 & 66.8 & 54.6 & 79.9 & 69.6 & 24.8 & 15.5 & 35.0 & 23.7 & 46.4 & 33.7 & 58.4 & 44.7 \\
\hline$V_{G P}$ & 35.8 & 25.4 & 52.0 & 40.1 & 67.8 & 56.3 & 80.6 & 71.5 & 25.7 & 16.2 & 36.1 & 24.9 & 48.0 & 35.1 & 59.7 & 46.7 \\
\hline$\widetilde{V}_{A I C}$ & 40.8 & 29.2 & 58.4 & 46.3 & 74.6 & 63.8 & 86.3 & 78.3 & 29.9 & 19.8 & 42.3 & 30.5 & 55.3 & 43.2 & 67.6 & 56.0 \\
\hline$V_{S C}$ & 39.5 & 28.0 & 56.7 & 44.4 & 72.8 & 61.4 & 84.7 & 76.2 & 27.6 & 17.7 & 38.9 & 26.7 & 50.7 & 37.3 & 62.6 & 49.0 \\
\hline & \multicolumn{16}{|c|}{ Nonlinear Moving Average } \\
\hline$V_{i d}$ & 18.5 & 10.0 & 25.3 & 14.6 & 34.5 & 20.6 & 44.4 & 28.3 & 16.2 & 9.2 & 21.8 & 12.5 & 28.9 & 17.2 & 37.3 & 23.4 \\
\hline$V^{*}$ & 23.1 & 14.4 & 32.5 & 22.0 & 43.1 & 31.3 & 54.4 & 41.7 & 19.3 & 11.5 & 26.2 & 16.9 & 34.7 & 23.6 & 43.6 & 31.4 \\
\hline${\underset{\sim}{G P}}_{G P}$ & 24.9 & 16.3 & 35.4 & 24.9 & 46.9 & 35.5 & 58.2 & 46.8 & 21.1 & 13.4 & 29.1 & 19.7 & 38.4 & 27.6 & 47.8 & 36.7 \\
\hline$V_{A I C}$ & 27.8 & 18.7 & 40.0 & 28.9 & 53.1 & 41.4 & 65.1 & 54.1 & 23.6 & 15.3 & 32.8 & 22.9 & 43.4 & 32.5 & 54.0 & 42.5 \\
\hline$\widetilde{V}_{S C}$ & 27.8 & 18.5 & 40.1 & 28.7 & 53.1 & 41.2 & 65.1 & 53.7 & 23.2 & 15.6 & 32.5 & 23.2 & 43.0 & 32.8 & 53.2 & 43.0 \\
\hline
\end{tabular}

Notes: The number of replications is 25,000 and $T=1024$. The critical values are adjusted so that empirical rejection probabilities of the tests under the null are exactly 0.1 and 0.05 for two-sided tests. The empirical powers are given in percentages. 
Table 6. Empirical Powers for an MA(2) process

\begin{tabular}{|c|c|c|c|c|c|c|c|c|c|c|c|c|c|c|c|c|}
\hline \multirow{3}{*}{$\rho_{1}=\rho_{2}$} & \multicolumn{8}{|c|}{$q=4$} & \multicolumn{8}{|c|}{$q=8$} \\
\hline & \multicolumn{2}{|c|}{0.040} & \multicolumn{2}{|c|}{0.055} & \multicolumn{2}{|c|}{0.070} & \multicolumn{2}{|c|}{0.085} & \multicolumn{2}{|c|}{0.040} & \multicolumn{2}{|c|}{0.055} & \multicolumn{2}{|c|}{0.070} & \multicolumn{2}{|c|}{0.085} \\
\hline & 10 & 5 & 10 & 5 & 10 & 5 & 10 & 5 & 10 & 5 & 10 & 5 & 10 & 5 & 10 & 5 \\
\hline & \multicolumn{16}{|c|}{$\operatorname{GARCH}(1,1)$ with normal errors } \\
\hline$V_{i d}$ & 40.8 & 28.4 & 59.1 & 45.9 & 75.5 & 63.9 & 87.1 & 78.8 & 40.8 & 28.4 & 59.1 & 45.9 & 75.5 & 63.9 & 87.1 & 78.8 \\
\hline$V^{*}$ & 41.0 & 29.1 & 59.1 & 46.5 & 75.0 & 64.1 & 86.2 & 78.4 & 41.0 & 29.1 & 59.1 & 46.5 & 75.0 & 64.1 & 86.2 & 78.4 \\
\hline$V_{G P}$ & 41.6 & 30.0 & 60.1 & 47.9 & 75.9 & 65.5 & 86.9 & 79.6 & 41.6 & 30.0 & 60.1 & 47.9 & 75.9 & 65.5 & 86.9 & 79.6 \\
\hline$\widetilde{V}_{A I C}$ & 43.5 & 31.5 & 62.2 & 50.1 & 77.6 & 67.7 & 88.2 & 81.1 & 43.5 & 31.5 & 62.2 & 50.1 & 77.6 & 67.7 & 88.2 & 81.1 \\
\hline$\widetilde{V}_{S C}$ & 42.8 & 31.0 & 61.4 & 49.4 & 77.0 & 67.0 & 87.7 & 80.6 & 42.8 & 31.0 & 61.4 & 49.4 & 77.0 & 67.0 & 87.7 & 80.6 \\
\hline & \multicolumn{16}{|c|}{ EGARCH(1,1) with normal errors } \\
\hline$V_{i d}$ & 16.3 & 8.4 & 22.9 & 11.9 & 31.1 & 17.2 & 41.0 & 24.3 & 16.3 & 8.4 & 22.9 & 11.9 & 31.1 & 17.2 & 41.0 & 24.3 \\
\hline$V^{*}$ & 19.6 & 11.7 & 27.8 & 18.0 & 37.7 & 26.0 & 48.2 & 35.8 & 19.6 & 11.7 & 27.8 & 18.0 & 37.7 & 26.0 & 48.2 & 35.8 \\
\hline$V_{G P}$ & 21.0 & 12.8 & 29.7 & 19.9 & 40.2 & 28.7 & 51.3 & 39.0 & 21.0 & 12.8 & 29.7 & 19.9 & 40.2 & 28.7 & 51.3 & 39.0 \\
\hline$\widetilde{V}_{A I C}$ & 23.3 & 15.5 & 33.5 & 23.9 & 45.2 & 34.5 & 56.8 & 46.0 & 23.3 & 15.5 & 33.5 & 23.9 & 45.2 & 34.5 & 56.8 & 46.0 \\
\hline$\widetilde{V}_{S C}$ & \multicolumn{16}{|c|}{ Bilinear } \\
\hline$V_{i d}$ & 30.0 & 19.4 & 45.0 & 32.1 & 60.9 & 47.2 & 74.9 & 62.7 & 30.0 & 19.4 & 45.0 & 32.1 & 60.9 & 47.2 & 74.9 & 62.7 \\
\hline$V^{*}$ & 30.4 & 20.5 & 45.4 & 33.6 & 61.6 & 49.0 & 75.5 & 64.8 & 30.4 & 20.5 & 45.4 & 33.6 & 61.6 & 49.0 & 75.5 & 64.8 \\
\hline$V_{G P}$ & 31.7 & 22.0 & 47.1 & 36.1 & 63.7 & 52.1 & 77.3 & 67.8 & 31.7 & 22.0 & 47.1 & 36.1 & 63.7 & 52.1 & 77.3 & 67.8 \\
\hline$\widetilde{V}_{A I C}$ & 34.7 & 23.8 & 51.6 & 39.2 & 68.1 & 56.5 & 81.1 & 72.0 & 34.7 & 23.8 & 51.6 & 39.2 & 68.1 & 56.5 & 81.1 & 72.0 \\
\hline$\widetilde{V}_{S C}$ & 33.8 & 23.1 & 50.3 & 37.8 & 66.5 & 54.8 & 79.9 & 70.3 & 33.8 & 23.1 & 50.3 & 37.8 & 66.5 & 54.8 & 79.9 & 70.3 \\
\hline & \multicolumn{16}{|c|}{ Nonlinear Moving Average } \\
\hline$V_{i d}$ & 17.1 & 9.1 & 23.6 & 13.5 & 32.1 & 19.4 & 41.8 & 26.6 & 17.1 & 9.1 & 23.6 & 13.5 & 32.1 & 19.4 & 41.8 & 26.6 \\
\hline$V^{*}$ & 21.7 & 13.7 & 31.3 & 21.5 & 41.9 & 31.1 & 52.8 & 41.5 & 21.7 & 13.7 & 31.3 & 21.5 & 41.9 & 31.1 & 52.8 & 41.5 \\
\hline$V_{G P}$ & 23.6 & 15.4 & 33.9 & 24.2 & 45.3 & 34.7 & 56.6 & 46.2 & 23.6 & 15.4 & 33.9 & 24.2 & 45.3 & 34.7 & 56.6 & 46.2 \\
\hline$\widetilde{V}_{A I C}$ & 24.2 & 15.5 & 34.8 & 24.6 & 46.5 & 35.3 & 58.1 & 46.4 & 24.2 & 15.5 & 34.8 & 24.6 & 46.5 & 35.3 & 58.1 & 46.4 \\
\hline$\widetilde{V}_{S C}$ & 24.3 & 15.4 & 34.9 & 24.4 & 46.6 & 35.0 & 58.0 & 46.4 & 24.3 & 15.4 & 34.9 & 24.4 & 46.6 & 35.0 & 58.0 & 46.4 \\
\hline
\end{tabular}

Notes: The number of replications is 25,000 and and $T=1024$. The critical values are adjusted so that empirical rejection probabilities of the tests under the null are exactly 0.1 and 0.05 for two-sided tests. The empirical powers are given in percentages. 